\title{
Induction of Superhydrophobicity in a Cellulose Substrate by LbL Assembly of Covalently Linked Dual-Sized Silica Nanoparticles Layers
}

\author{
Chenghua Yu1, Feng Wang1, Lucian A. Lucia², Shiyu Fu ${ }^{1^{*}}$ \\ ${ }^{1}$ State Key Laboratory of Pulp \& Paper Engineering, South China University of Technology, Guangzhou, China \\ ${ }^{2}$ Department of Forest Biomaterials, North Carolina State University, Raleigh, NC, USA \\ Email: ^shyfu@scut.edu.cn
}

How to cite this paper: Yu, C.H., Wang, F., Lucia, L.A. and Fu, S.Y. (2017) Induction of Superhydrophobicity in a Cellulose Substrate by LbL Assembly of Covalently Linked Dual-Sized Silica Nanoparticles Layers. Advances in Materials Physics and Chemistry, 7, 395-410.

https://doi.org/10.4236/ampc.2017.712031

Received: November 22, 2017

Accepted: December 24, 2017

Published: December 27, 2017

Copyright ( $\odot 2017$ by authors and Scientific Research Publishing Inc. This work is licensed under the Creative Commons Attribution International License (CC BY 4.0).

http://creativecommons.org/licenses/by/4.0/

\begin{abstract}
Micro/nano texturized oxidized cellulose membranes (MNOCM) were constructed by layer-by-layer (LbL) assembly in which a base cellulose film was modified by covalent linkages to amino-functionalized silica nanoparticles (amino-SiO $\mathrm{SP}_{2} \mathrm{NPs}, 260 \mathrm{~nm}$ diameter) and epoxy-functionalized silica nanoparticles (epoxy- $\mathrm{SiO}_{2} \mathrm{NPs}, 30 \mathrm{~nm}$ diameter). The amino- $\mathrm{SiO}_{2} \mathrm{NPs}$ grafted onto the MNOCM surface through a standard amidation reaction between the amino groups of the $\mathrm{SiO}_{2}$ NPs and the carboxyl groups of the MNOCM surface in the presence of EDC and NHS consequently forming a first layer of large $(260 \mathrm{~nm})$ nanoparticles; subsequently, it was reacted with smaller (30 $\mathrm{nm}$ ) epoxy- $\mathrm{SiO}_{2} \mathrm{NPs}$. Continuous repetitions of these alternating sized silica NPs through a standard LbL approach lead to a highly micro/nano-texturized MNOCM film as shown by SEM, which was ultimately sealed with a layer of hydrophobic PFOTES $(1 \mathrm{H}, 1 \mathrm{H}, 2 \mathrm{H}, 2 \mathrm{H}$-perfluorooctyltriethoxysilane). Although the wettability of MNOCM was no longer hydrophilic, it was found that at five layers deep of NPs, it became superhydrophobic as evidenced by a water contact angle of $151^{\circ} \pm 2^{\circ}$ and slide angle of $4^{\circ}$. The change in wettability was attributed to increases in final LbL layer surface roughness induced by the sufficient LbL layering of alternating sizes of NPs akin to what is observed in a lotus leaf surface. It was also noted that these superhydrophobic-MNOCM materials displayed good self-cleaning.
\end{abstract}

\section{Keywords}

Superhydrophobic, Cellulose Membranes, Layer-by-Layer Assembly

\section{Introduction}

Materials that display superhydrophobicity may demonstrate the unique property 
of self-cleaning as typified by a pristine lotus leaf surrounded by mud. It has been determined that the surface of such materials possess a hierarchical dual-size roughness and low surface energy [1]; thus, control of surface roughness and low surface energy via chemical composition are the two principal motifs to induce superhydrophobicity [2] [3] [4] [5]. A number of methods have emerged to endow hierarchical dual-size roughness structured surfaces, including solution-immersion process [6] [7], electrospinning [8], chemical vapor deposition [9] [10], and layer-by-layer assembly (LbL) [2] [5] [11] [12] [13]. LbL is popular among these methods to imbue stable rough surfaces on various sizes of silica particles especially via continuous covalent bonding of functionalized silica nanoparticles instead of using charged polymers to conglutinate the particles. Guittard et al. [2] found that the hydrophobicity increased with the number of layers and the static contact angle with water could reach $150^{\circ} \pm 3^{\circ}$ and the contact angle hysteresis could reach $12^{\circ}$ with the alternation of nine layers.

In general, such silica particle-based superhydrophobic materials are based on hard base substrates such as silicon, glass, or metals. Recently, however, the development of soft superhydrophobic materials based on cellulose including textiles [5] [14] [15] and paper [16] is attractive due to its low-cost, biodegradability, renewability and environmental friendliness. However, NPs attached to paper substrates possess no intrinsic stability and detach from the substrate when applied. The objective of this work was to prepare a stable substrate membrane hosting a suite of carboxyl groups on the surface to provide active sites for NPs and using inspiration from nature (the lotus leaf) thereby construct a dual NP-sized architecturally rough structure.

The primary hydroxyl group on the C- 6 of glucose in cellulose can be selectively oxidized to carboxylate groups with 2,2,6,6-tetramethyl-1-piperidinyloxy (TEMPO)-mediated oxidation [17] [18] [19] [20] [21] that can couple amino group from amino-functionalized polymers through carbodiimide-mediated reactions [22]. An added bonus is that the intrinsic wet strength of TEMPO-oxidized cellulose membranes is significantly enhanced [23] [24]. However, compared to plastic, TEMPO-oxidized cellulose is highly hydrophilic and cannot meet the requirement of high hydrophobicity for many applications.

Herein, we present a facile and direct method to produce TEMPO-oxidized cellulose-based superhydrophobic membranes characterized by covalent layer-bylayer assembly of dual-sized nanoparticles. The cellulose fibers were first oxidized by TEMPO-mediated oxidation to form carboxylate groups followed by coupling to the amino group of large amino- $\mathrm{SiO}_{2} \mathrm{NPs}$ through carbodiimide-mediated reactions. Later, smaller (almost ten times) epoxy- $\mathrm{SiO}_{2} \mathrm{NPs}$ were deposited onto the amino- $\mathrm{SiO}_{2} \mathrm{NPs}$ to form double-layered silica NPs and thus provide a micro/nano texturized cellulose membrane (MNOCM). Lastly, it was capped with chemical vapor deposition (CVD) to (1) provide a capping monolayer, (2) remove any wettability possible from the Si NPs, and (3) thereby test the effect of dual-sized NP roughness on induced hydrophobicity changes on the 
cellulose membrane. The obtained MNOCM films showed not only superhydrophobicity, but self-cleaning.

\section{Experimental}

\subsection{Materials}

The bleached softwood kraft pulps (BSKP) with a moisture content of $8 \%$ were provided by Guangzhou Paper Mill. 3-Glycidyloxypropyltrimethoxysilane (GPS), (3-Aminopropyl) tri-ethoxy-silane (AMS), Silicon dioxide $(30 \mathrm{~nm})$, $\mathrm{N}$-(3-Dimethylaminopropyl)-N'-ethyl-carbodiimide hydrochloride (EDC), NHydroxy-succinimide (NHS) and $1 \mathrm{H}, 1 \mathrm{H}, 2 \mathrm{H}, 2 \mathrm{H}$-perfluorooctyltriethoxysilane (PFOTES) were all purchased from Aladdin Chemistry Co., Ltd. (Guangzhou, China). Tetraethyl orthosilicate (TEOS), ammonium hydroxide ( $30 \%$ in water), and all other chemicals were purchased by Tianjin Chemical Co., Ltd. (Tianjin, China).

\subsection{Preparation of TEMPO-Oxidized Cellulose Membranes (MNOCM)}

BSKP (10 g, dry weight) was dispersed into $1 \mathrm{~L}$ of deionized water with continuous mechanical stirring at $300 \mathrm{rpms}$, followed by addition of $\mathrm{NaBr}(0.1 \mathrm{~g} / \mathrm{g}$ d.w.p.) and TEMPO (0.015 g/g d.w.p.) [25]. The reaction was started by addition of $\mathrm{NaClO}$ solution $(12 \%, 6,8$ and $10 \mathrm{mmol} / \mathrm{g}$ d.w.p. $)$ at $25^{\circ} \mathrm{C}$. $\mathrm{pH}$ of the reaction was maintained at $10 \pm 0.2$ by addition of $0.5 \mathrm{M} \mathrm{NaOH}$ until no further decrease. Finally, the pulp was washed with deionized water and filtered by a Büchner funnel with a filter cloth (400-mesh) to obtain TEMPO-oxidized cellulose (OC) suspension at a concentration of $0.5 \mathrm{wt} \%$.

TEMPO-oxidized cellulose membranes (MNOCM) were prepared by vacuum filtering $50 \mathrm{ml}$ of TEMP-oxidized cellulose suspension on a $0.45 \mu \mathrm{m}$ filter membrane and dried at room temperature.

\subsection{Preparation of Amino-Functionalized Silica Nanoparticles (Amino- $\mathrm{SiO}_{2} \mathrm{NPs}$ )}

A variety of sizes of silica nanoparticles were obtained by polymerization of TEOS using the Stöber method [26]. Typically, a fixed volume of TEOS was added drop wise to a flask under magnetic stirring containing ammonia solution, water, and ethanol (see Table 1) followed by addition of $5 \mathrm{~mL}$ of APS and heating at $70^{\circ} \mathrm{C}$ for $6 \mathrm{~h}$. The resulting silica nanoparticles were separated by centrifugation and washed by ethanol in triplicate. Finally, the obtained particle was dried in vacuo at $50^{\circ} \mathrm{C}$ overnight.

\subsection{Preparation of Epoxy-Functionalized Silica Nanoparticles (Epoxy-SiO 2 NPs)}

$2 \mathrm{~g}$ of silica nanoparticles of $30 \mathrm{~nm}$ diameter were dispersed into $70 \mathrm{~mL}$ anhydrous toluene and ultrasonicated for 10 mins. Subsequently, $7 \mathrm{~mL}$ of GPS was 
Table 1. Preparation of silica nanoparticles.

\begin{tabular}{ccccccc}
\hline $\begin{array}{c}\text { Size } \\
(\mathrm{nm})\end{array}$ & $\begin{array}{c}\mathrm{TEOS} \\
(\mathrm{mL})\end{array}$ & $\begin{array}{c}\mathrm{NH}_{3} \mathrm{H}_{2} \mathrm{O} \\
(\mathrm{mL})\end{array}$ & $\begin{array}{c}\text { Ethanol } \\
(\mathrm{mL})\end{array}$ & $\begin{array}{c}\mathrm{H}_{2} \mathrm{O} \\
(\mathrm{mL})\end{array}$ & $\begin{array}{c}\text { Temperature } \\
\left({ }^{\circ} \mathrm{C}\right)\end{array}$ & $\begin{array}{c}\text { Time } \\
(\mathrm{h})\end{array}$ \\
\hline $70[27]$ & 13.2 & 33 & 440 & - & 60 & 5 \\
$260[12]$ & 13.2 & 33 & 440 & 8.8 & $\mathrm{RT}$ & 17 \\
$500[28]$ & 10 & 22 & 100 & - & $\mathrm{RT}$ & 8 \\
\hline
\end{tabular}

added drop wise with mechanical stirring, and was kept at $126^{\circ} \mathrm{C}$ under $\mathrm{N}_{2}$ atmosphere for $3 \mathrm{~h}$. The resulting silica nanoparticles were separated by centrifugation and washed with toluene in triplicate. Finally, the epoxy-functionalized silica nanoparticles were dried in vacuo at $50^{\circ} \mathrm{C}$ overnight.

\subsection{Covalent LbL Assembly to Derive the Micro/Nano Surface Texture}

Micro/nano surface architecture was obtained by inter-grafting the two sizes of nanoparticles as obtained from the LbL assembly process. The first step was grafting amino- $\mathrm{SiO}_{2} \mathrm{NPs}$ onto the MNOCM through carbodiimide-mediated coupling of the amino group from amino- $\mathrm{SiO}_{2} \mathrm{NPs}$ to the carboxyl group of the TEMPO-oxidized cellulose membranes. First, $0.02 \mathrm{~g}$ of AS was dispersed into 8 $\mathrm{mL}$ of ethanol and ultrasonicated for 10 mins. Subsequently, $0.1 \mathrm{~g}$ EDC, $0.1 \mathrm{~g}$ NHS, and MNOCM were added and kept at room temperature for $4 \mathrm{~h}$. After reaction, the MNOCM@SiO $2-\mathrm{NH}_{2}$ was removed and washed with ethanol for three times.

The second step was reacting $\mathrm{MNOCM} @ \mathrm{SiO}_{2}-\mathrm{NH}_{2}$ and epoxy-SiO${ }_{2} \mathrm{NPs} .0 .02$ $\mathrm{g}$ of epoxy- $\mathrm{SiO}_{2} \mathrm{NPs}$ by dispersing into $8 \mathrm{~mL}$ of ethanol and ultrasonicating for 10 mins. Then the MNOCM@SiO $2-\mathrm{NH}_{2}$ was immersed in the solution at $40^{\circ} \mathrm{C}$ for $12 \mathrm{~h}$ to afford surface 1 (denoted as 1 layer). Several cycles were repeated to afford 2 to 5 additional layers.

\subsection{Silanization of MNOCM Using Chemical Vapor Deposition (CVD)}

The as-prepared MNOCMs were treated with PFOTES by chemical vapor phase deposition [9]. The samples were placed in a $250 \mathrm{~mL}$ metal bottle containing two small open bottles holding $200 \mu \mathrm{L}$ of PFOTES and $1 \mathrm{~mL}$ of deionized water. The metal bottle was then sealed and heated inside an $80^{\circ} \mathrm{C}$ oven for $6 \mathrm{~h}$. Subsequently, the surface-treated samples were taken out and placed in a vacuum oven for more than $1 \mathrm{~h}$ at $60^{\circ} \mathrm{C}$ to remove unreacted silane.

\subsection{Transmission Electron Microscopy (TEM)}

The size distribution of $\mathrm{SiO}_{2}$ NPs was obtained from TEM images. Samples were dispersed in ethanol $(0.02 \% \mathrm{w} / \mathrm{v})$ and deposited on a carbon-coated copper grid. The images were observed by JEM 2100 transmission electron microscope at an accelerating voltage of $200 \mathrm{kV}$. 


\subsection{Morphology and Nanostructure}

The MNOCM were first sectioned into pieces. These pieces were fixed on sample holders and coated with Au for SEM (Merlin, Zeiss, Germany) at an operating voltage of $10 \mathrm{kV}$ in secondary electron mode.

\subsection{Test of ATR-Fourier Transform-Infrared (ATR-FT-IR) Spectroscopy}

ATR-FT-IR spectra were recorded on a VERTEX 70 infrared spectrum instrument (Bruker Corporation, Germany). The scanning range was $500-4000 \mathrm{~cm}^{-1}$.

\subsection{Contact Angle}

Water contact angle (WCA) measurements were carried out on an OCA20 Micro (Data physics Instruments) to evaluate hydrophobicities by using a droplet $(5 \mu \mathrm{L})$ of deionized water as an indicator at room temperature. The values reported were taken after the contact angle equilibrated.

\section{Results and Discussion}

\subsection{Preparation of Silica Nanoparticles}

In general, the pre-eminent physical factors that contribute to the phenomenon referred to as superhydrophobicity are (1) roughness or surface texture and (2) attenuated (low) surface energy [29]. The silica nanoparticles to induce the micro/nano texturized structure on the MNOCM surface were prepared by the Stober procedure which is characterized by catalyzed hydrolysis and condensation of TEOS under alkaline conditions [12] [30] (Figure 1(a)). It is noteworthy that in the process of TEOS hydrolysis, the ammonia content, alcohol dosage, temperature and the time of the reaction influence the sizes of silica nanoparticles.

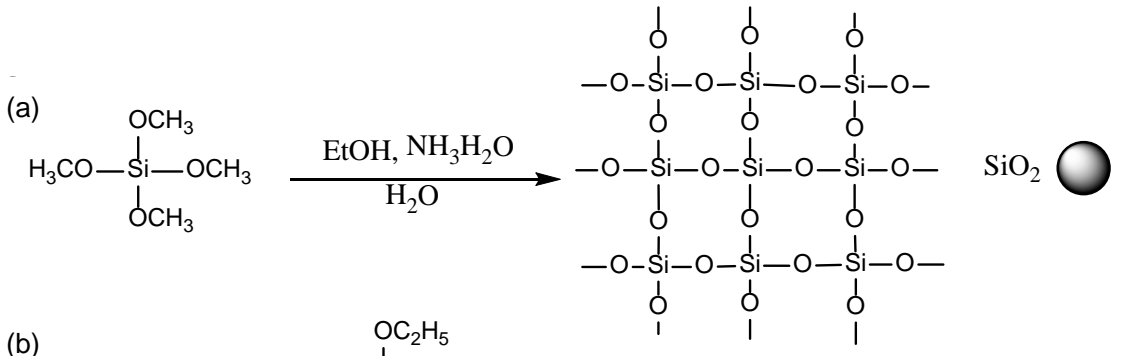

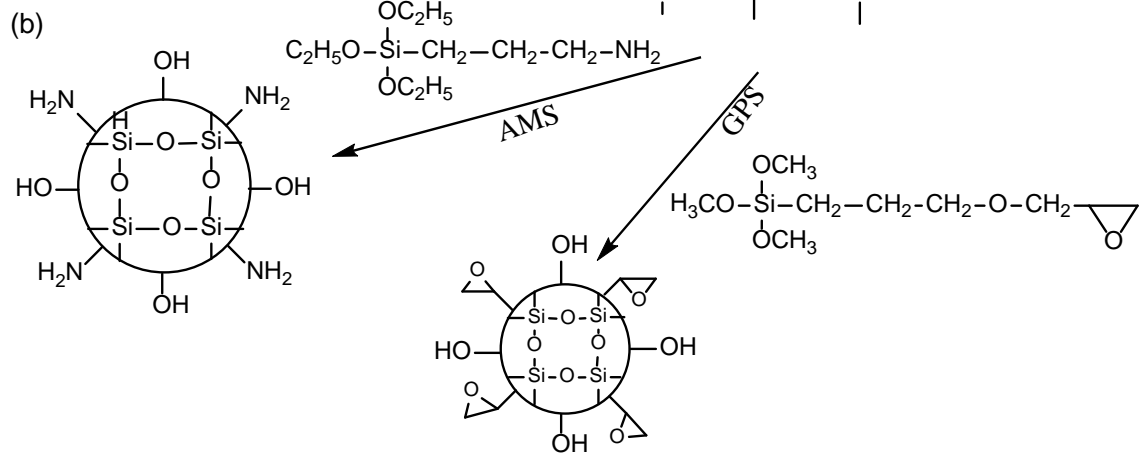

Figure 1. Scheme for the preparation of silica nanoparticles. 

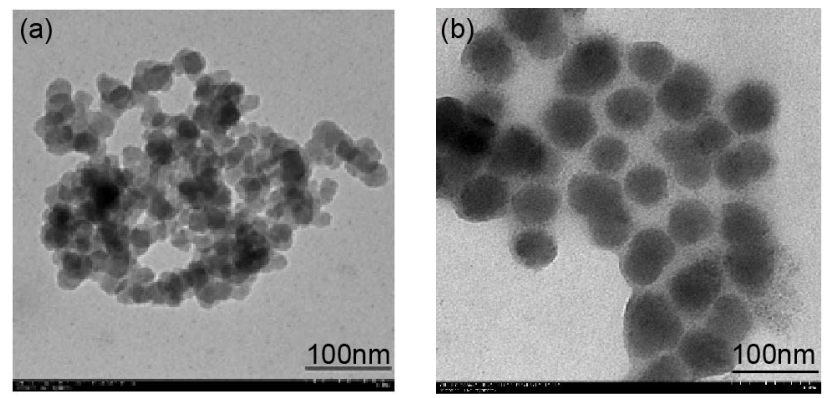

A variety of multi-sized silica nanoparticles were obtained by changing the reaction conditions (Table 1). The formation of a stable interaction between the MNOCM surface and silica nanoparticles was accomplished by grafting these silica nanoparticle via their active sites. In the present work, AMS-functionalized silica nanoparticles (amino- $\mathrm{SiO}_{2} \mathrm{NPs}$ ) and GPS functionalized silica nanoparticles (epoxy-SiO${ }_{2} \mathrm{NPs}$ ) were prepared by hydrolytic condensation of AMS or GPS with $\mathrm{SiO}_{2}$, respectively (Figure 1(b)). The AMS or GPS was hydrolyzed and grafted onto the silica nanoparticles surface to afford surface reactive amino groups or epoxy groups.

Morphologies and nanoparticle sizes of the as-prepared $\mathrm{SiO}_{2} \mathrm{NPs}$ are shown in Figure 2. The TEMs showed that the sizes of the obtained silica nanoparticles increased after sequential changes in reaction conditions. The $\mathrm{SiO}_{2}$ nanoparticles presented spherical shapes with an average diameter of $\sim 30 \mathrm{~nm}, \sim 70 \mathrm{~nm}, \sim 260$ $\mathrm{nm}$ and $\sim 500 \mathrm{~nm}$. In Figure 3, all the silica nanoparticles exhibited strong bands at $\sim 800 \mathrm{~cm}^{-1}$ and $\sim 1100 \mathrm{~cm}^{-1}$ corresponding to the Si-O-Si asymmetric and symmetric stretching vibrations, respectively [31]. The peaks at $\sim 3421 \mathrm{~cm}^{-1}$ and

Figure 2. TEM images of (a) $30 \mathrm{~nm} \mathrm{SiO}_{2}$, (b) $70 \mathrm{~nm} \mathrm{SiO}_{2}$, (c) $260 \mathrm{~nm} \mathrm{SiO}_{2}$ and (d) $500 \mathrm{~nm} \mathrm{SiO}_{2}$.
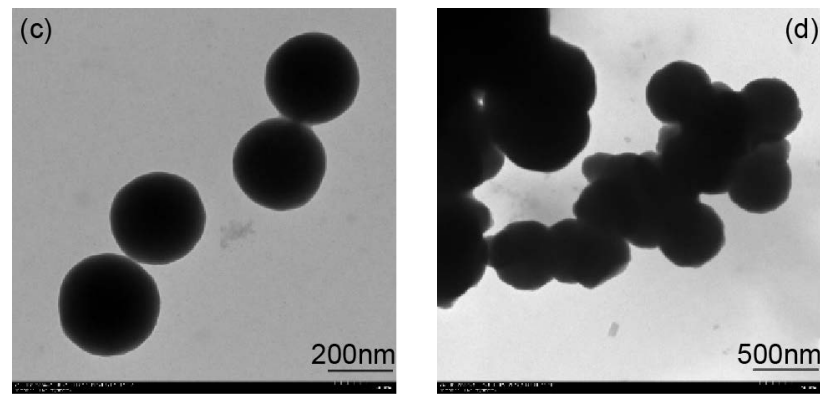

(d)

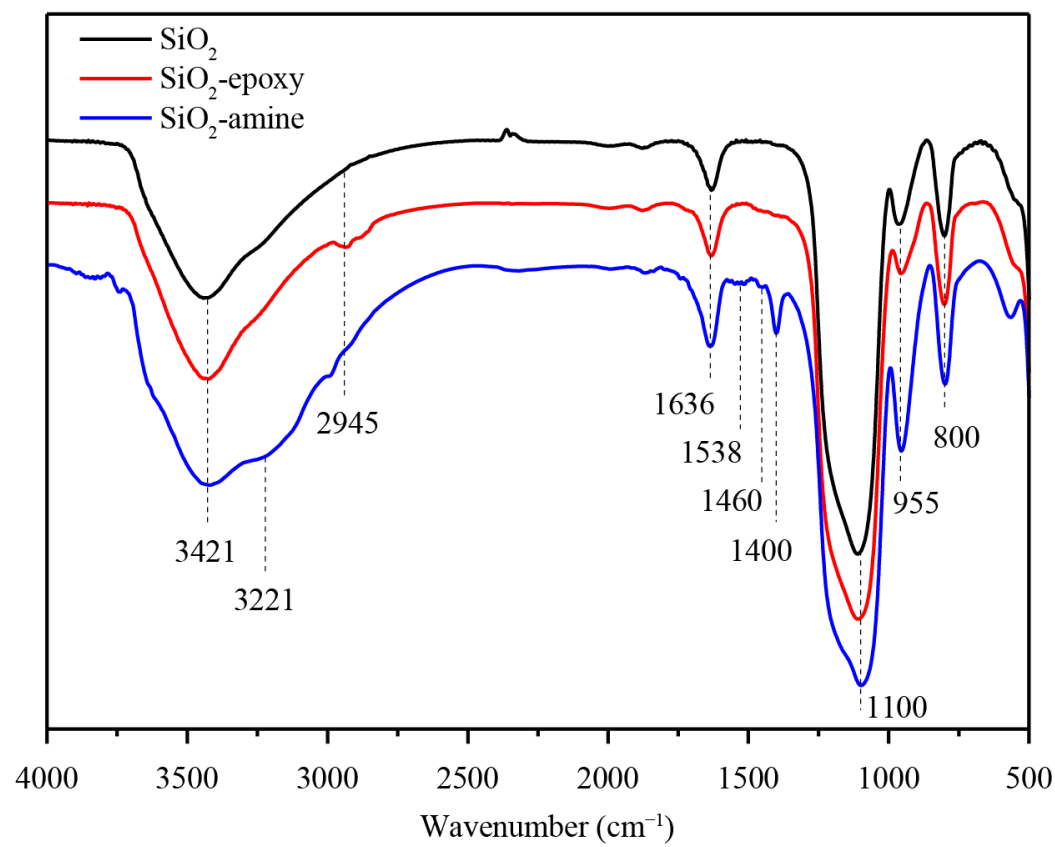

Figure 3. FT-IR spectra of silica nanoparticles before (after Stöber modification) and after AMS and GPS surface grafting. 
$1630 \mathrm{~cm}^{-1}$ belong to $\mathrm{O}-\mathrm{H}$ stretching and distorting vibrations on silica particles, respectively [32], whereas the peak at $\sim 955 \mathrm{~cm}^{-1}$ corresponded to the bending vibration of $\mathrm{Si}-\mathrm{OH}[11]$.

When the surface silica nanoparticles were grafted subsequently AMS or GPS, new peak appeared at $\sim 2945 \mathrm{~cm}^{-1}$, corresponds to the C-H stretching of $-\mathrm{CH}_{2}$ groups [11], which confirmed that AMS or GPS had been successfully grafted because both groups contain $-\mathrm{CH}_{2}-$. Moreover, the peaks (blue line) at $\sim 1538$ $\mathrm{cm}^{-1}$ ( $\mathrm{N}-\mathrm{H}$ bending) and $\sim 1400 \mathrm{~cm}^{-1}$ (C-H bending) further proved amino groups existed in the modified silica particles. The color of these modified particles changed blue-purple within a few min when added into 5\% ninhydrin aqueous solution. This reaction is the typical method to identify the amino group existence.

\subsection{Installation of NPs on the Surface of Oxidized Cellulose Membranes (OCM)}

In general, the rough micro/nano surface texture of the final MNOCM was created by covalent LbL assemblies of alternating large silica amino-NPs and smaller silica epoxy-functionalized-NPs. Figure 4 showed the scheme of reaction. TEMPO-mediated oxidation was carried out to produce varying levels of surface carboxyl on cellulose. The acid enriched sites were exploited to chemically anchor amino groups on the amino-SiO ${ }_{2} \mathrm{NPs}$ by activation with EDC and NHS (Figure 3). Additionally, free amino groups can react with the epoxy groups of epoxy-SiO ${ }_{2} \mathrm{NPs}$ by ring opening to afford layer 1 [2]. Subsequently, free epoxy groups can react with amino- $\mathrm{SiO}_{2} \mathrm{NPs}$ again. These reactions can be repeated as many times as desired to form $n$ layers. Finally, samples were treated with PFOTES through vapor phase deposition to provide a surface capping layer as stated previously (vide supra).
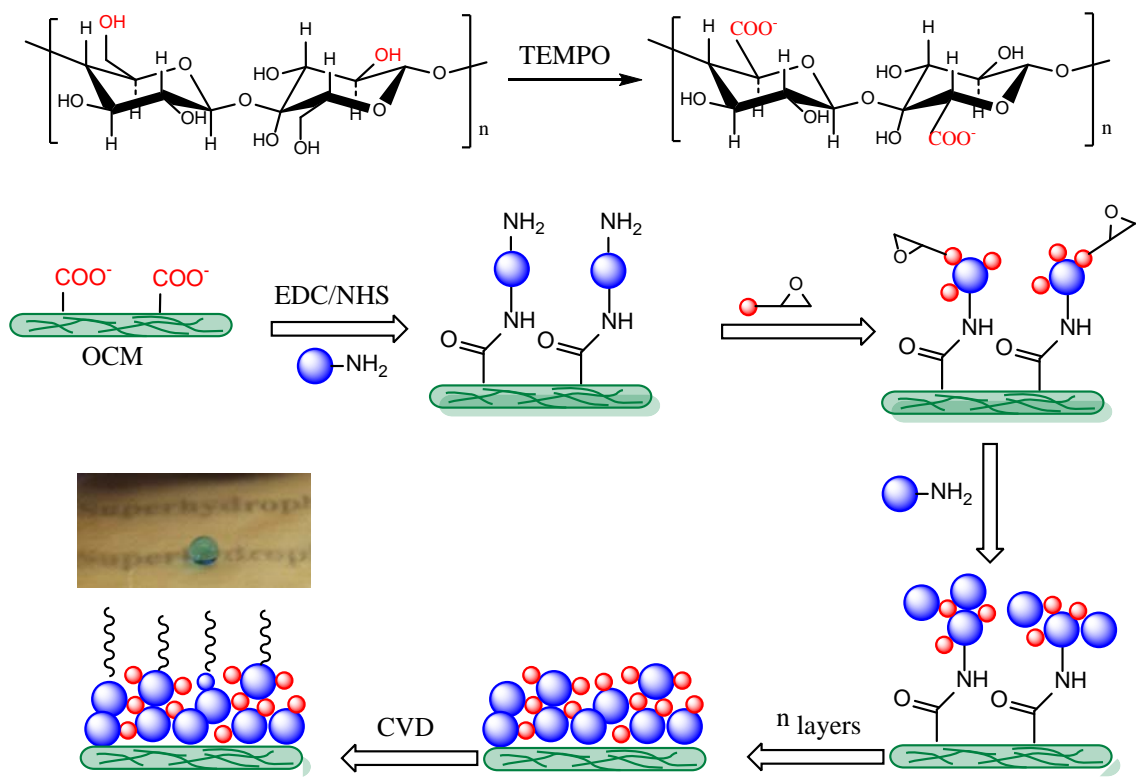

Figure 4. Schema of constructing rough micro/nano texture by covalent LbL assemblies. 
ATR-FT-IR (Figure 5) showed the ATR-FT-IR spectra of MNOCM and MNOCM@SiO ${ }_{2}-\mathrm{NH}_{2}$. The two samples exhibited strong absorbing peaks at $\sim 3335 \mathrm{~cm}^{-1}$ due to $\mathrm{O}-\mathrm{H}$ stretching of cellulose molecules. The peak at $\sim 2920$ $\mathrm{cm}^{-1}$ was attributed to $\mathrm{C}-\mathrm{H}$ stretching in $-\mathrm{CH}_{2}$ or $-\mathrm{CH}_{3}$ groups because the silica particles grafted $-\mathrm{CH}_{2}$ - groups. Meanwhile, the peaks at $\sim 1736 \mathrm{~cm}^{-1}$ and 1600 $\mathrm{cm}^{-1}$ could be attributed to the $-\mathrm{C}=\mathrm{O}$ and sodium carboxyl groups (-COONa), respectively [33]. Compared with $\mathrm{MNOCM}, \mathrm{MNOCM} @ \mathrm{SiO}_{2}-\mathrm{NH}_{2}$ had new peaks at $1698 \mathrm{~cm}^{-1}, 1544 \mathrm{~cm}^{-1}$ and $1279 \mathrm{~cm}^{-1}$, corresponding to the $\mathrm{C}=\mathrm{O}$ stretching vibration of -NHCO- (amide I), N-H bending (amide) and amide III, respectively [34] [35] [36] [37]. Moreover, two broad and enhanced peaks at 1050 $\mathrm{cm}^{-1}$ and $1029 \mathrm{~cm}^{-1}$ were characteristic of the overlapping absorption peaks between $\mathrm{Si}-\mathrm{O}-\mathrm{Si}$ and C-O-C [38]. The information confirmed that the amino- $\mathrm{SiO}_{2}$ NPs were successfully grafted onto the MNOCM surface through amino-carboxyl amidation [39].

Furthermore, the carboxyl content may influence grafting of amino- $\mathrm{SiO}_{2} \mathrm{NPs}$. Therefore, cellulose with different carboxyl contents $(0.95,1.24$, and 1.28 $\mathrm{mmol} / \mathrm{g}$ o.d.p) were prepared. It was found that the carboxyl content affected on the grafting of amino- $\mathrm{SiO}_{2}$ NPs showed in Figure 6. For the MNOCM with a carboxyl content $0.95 \mathrm{mmol} / \mathrm{g}$ o.d.p (Figure $6(\mathrm{a})$ ), we can clearly observe the surface was a little corrugated and smooth without any silica. Similar results can be observed in MNOCM with a carboxyl content $1.24 \mathrm{mmol} / \mathrm{g}$ o.d.p and 1.28 mmol/g o.d.p. For MNOCM@ $\mathrm{SiO}_{2}-\mathrm{NH}_{2}$ with a carboxyl content $0.95 \mathrm{mmol} / \mathrm{g}$ o.d.p (Figure 6(b)), the particles do not homogeneously over the surface and some places were exposed. The surface of MNOCM@ $\mathrm{SiO}_{2}-\mathrm{NH}_{2}$ were more covered by particles when the carboxyl content of MNOCM was $1.24 \mathrm{mmol} / \mathrm{g}$ o.d.p (Figure 6(c)), however, some areas were still exposed without particles. When

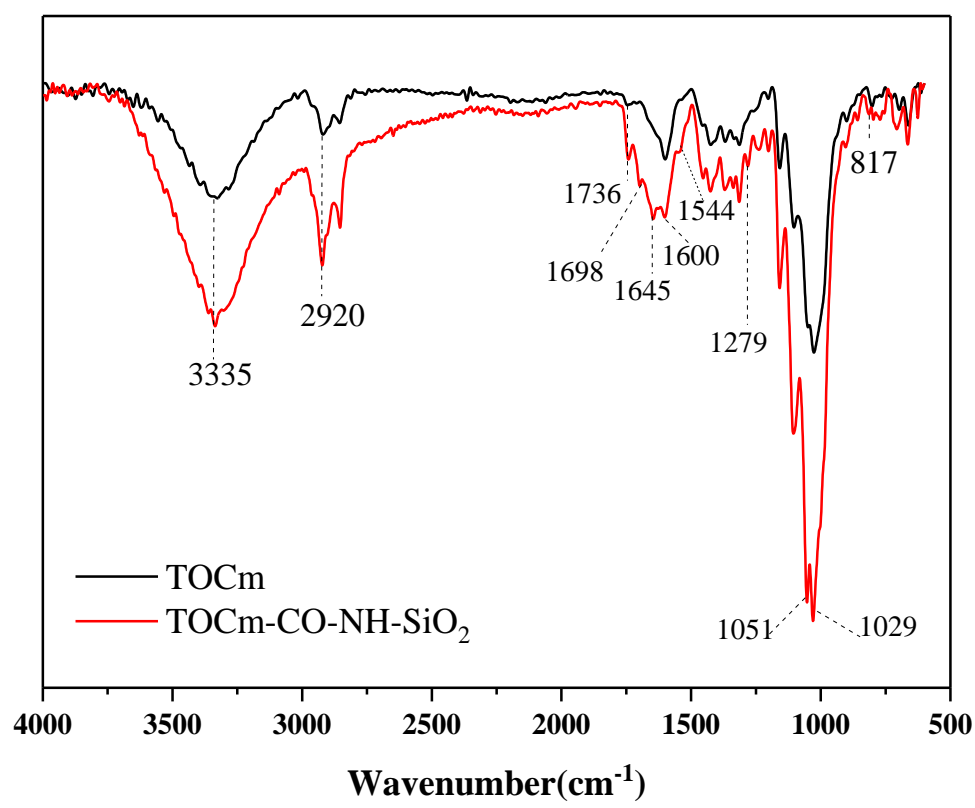

Figure 5. ATR-FT-IR spectra of MNOCM and MNOCM@ $\mathrm{SiO}_{2}-\mathrm{NH}_{2}$. 

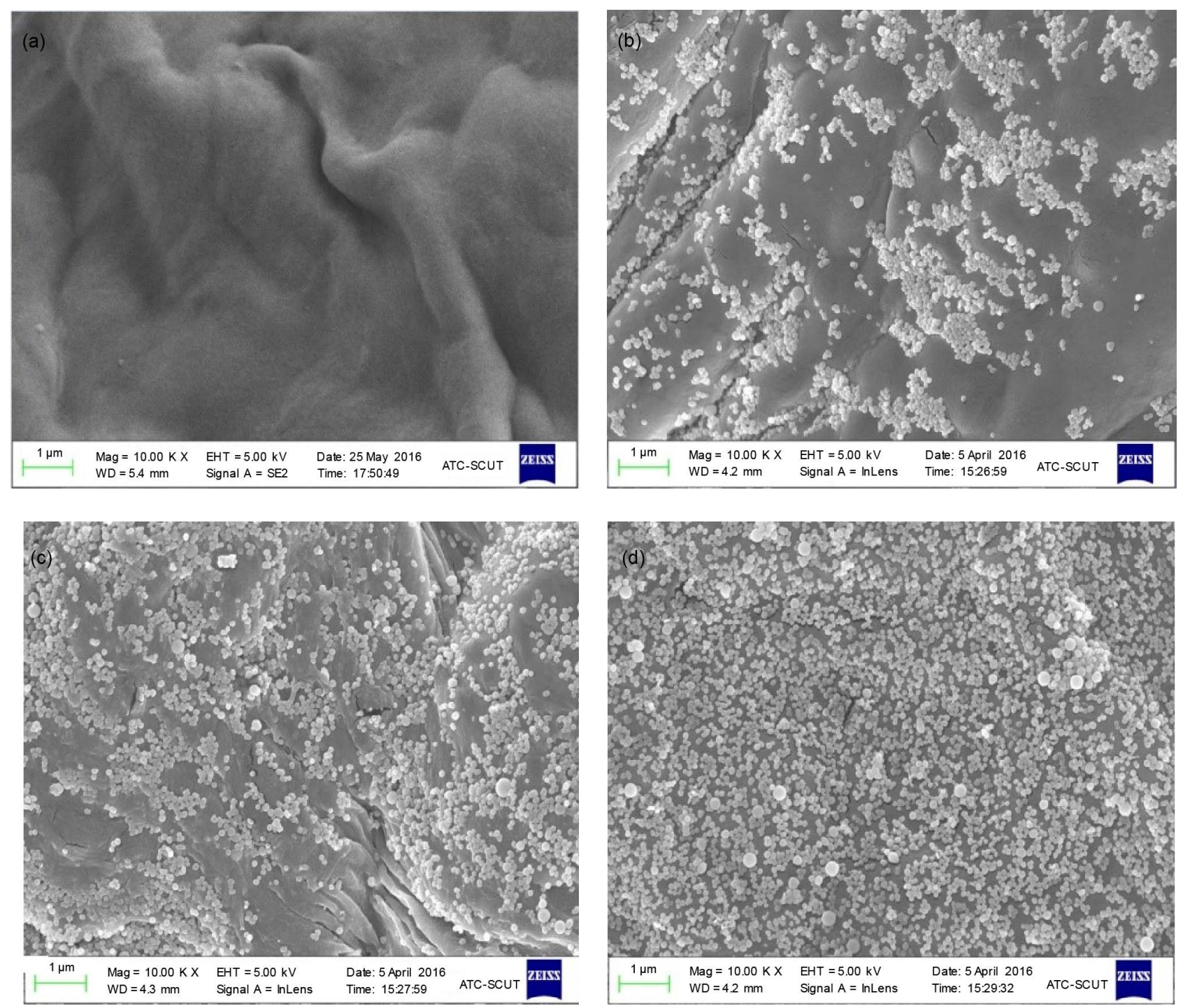

Figure 6. SEM images of cellulose films surfaced onto which $\mathrm{SiO}_{2}-\mathrm{NPs}$ are installed.

the MNOCM with a higher carboxyl content $(1.28 \mathrm{mmol} / \mathrm{g}$ o.d.p, Figure $6(\mathrm{~d}))$, more nanoparticles adhered to the surface randomly, almost completely covered. It revealed that the amount of silica on cellulose membranes surface increased with increasing carboxyl content.

To provide roughness to the MNOCM surface, $260 \mathrm{~nm}$ amino- $\mathrm{SiO}_{2} \mathrm{NPs}$ were grafted followed by covalent LbL assembly of $30 \mathrm{~nm} \mathrm{SiO}_{2} \mathrm{NPs}$. This was repeated several times to form $n$ layers of particles $(260 \mathrm{~nm}$ particles $+30 \mathrm{~nm}$ particles represented one layer). The SEM images in Figures 7(a)-7(e) showed the surface morphology of the MNOCM having a varying number of layers. The particles were randomly assembled indicating different degrees of surface roughness. For 1 layer (Figure 7(a)), the MNOCM surface was mainly covered with $260 \mathrm{~nm}$ particles and only a small amount of $30 \mathrm{~nm}$ particles, the average roughness is around $260 \mathrm{~nm}$. The roughness increased visually after 2 layers (Figure 7(b)). Figure 7(c) and Figure 7(d) showed the particle distribution in 

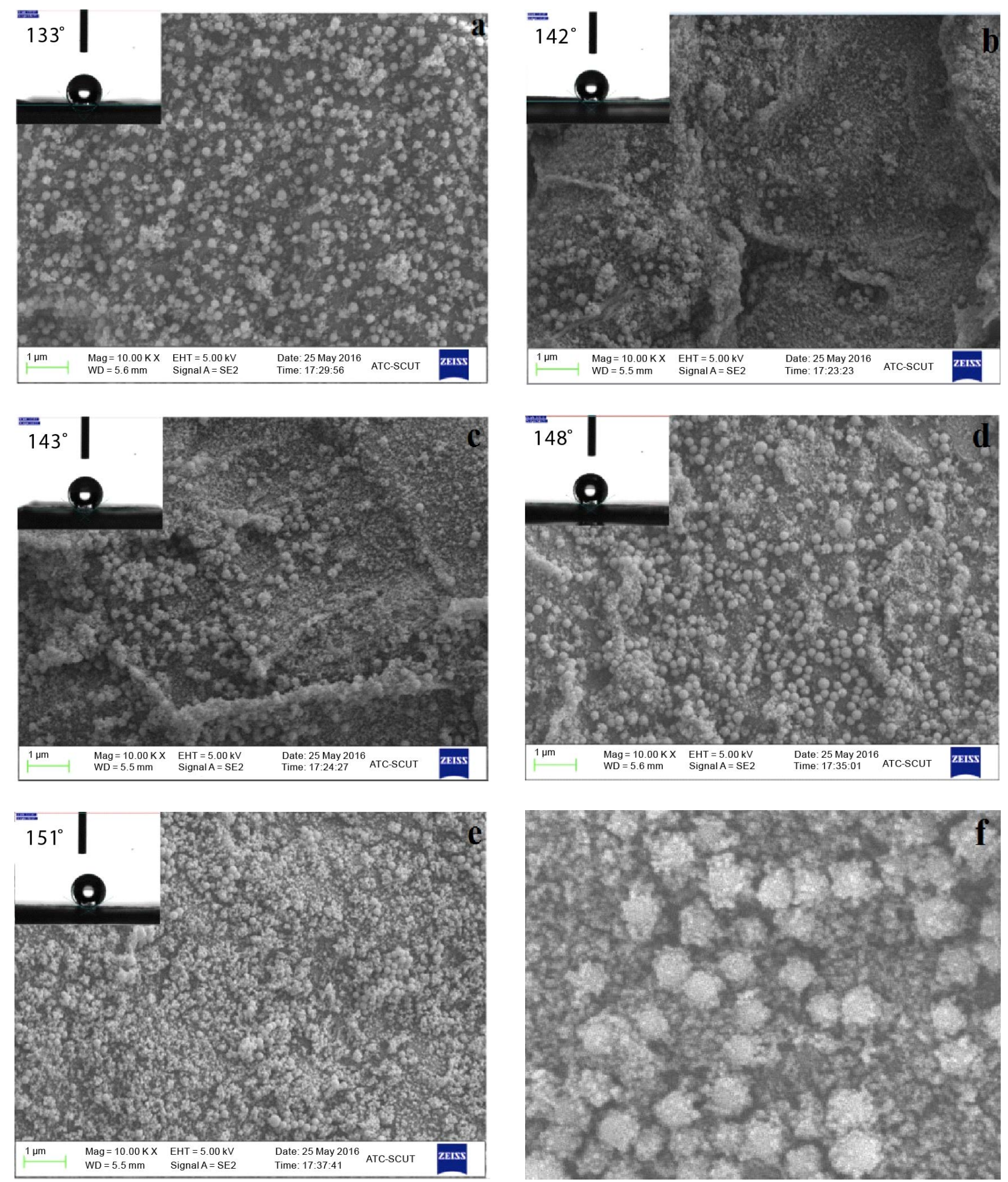

Figure 7. SEM images of MNOCM with (a) 1 layer, (b) 2 layers, (c) 3 layers, (d) 4 layers, (e) 5 layers, and (f) local enlarged image of 5 layers. The insets represent contact angle with water on the surface.

the 3 and 4 layers to form a rougher surface. Not surprisingly, after final assembly, a heavily rough surface was formed. The degree of roughness changed with each succeeding layer allowing for an elegant means of control of surface 
roughness through succeeding nanoparticles coverage [12] [40]. Interestingly, assembly of $30 \mathrm{~nm} \mathrm{SiO}{ }_{2} \mathrm{NPs}$ on the surface of $260 \mathrm{~nm} \mathrm{SiO}{ }_{2} \mathrm{NPs}$ allowed for the development of a raspberry-like structure (Figure $7(\mathrm{e})$, Figure $7(\mathrm{f})$ ) very evident in the 5 layers. The roughness of the surface after assembling from 1 layer to 5 layers doesn't increase linearly with the growth of layers because the small nanoparticles may plug the valley between particles. The suitable layers may provide best roughness structure, but not the biggest roughness.

The samples were subsequently capped with PFOTES by CVD and tested for wettability (Figure 7 insets). Not surprisingly, owing to its polyhydroxylic nature, pure OCM can be completely wetted by water. However, even after the deposition of one layer of NPs, the contact angle was $133^{\circ}$ (Figure 7(a)-inset). The pure OCM without particle covering is of course not superhydrophobic even capping with PFOTES. After deposition of 2 - 5 layers of particles (Figures 7 (b)-7(d) insets), the water contact angles increased to $142^{\circ}, 143^{\circ}, 148^{\circ}$, and ultimately $151^{\circ}$, respectively, with a slide angle of $4^{\circ}$ (Figure 8), demonstrating the attainment of a superhydrophobic surface. Such a change in contact angles indicated that the hydrophobicity of MNOCM surface displayed a hysteresis associated with LbL. Modifying the layers of nanoparticles can therefore effectively hydrophobize MNOCM.

The EDX of MNOCM after treatment with PFOTES is presented in Figure 9. Only carbon, oxygen, fluorine, silicon and sodium elements were detected. The carbon and oxygen were of course from MNOCM, silica, and PFOTES. Sodium was the counterion from the carboxyl groups to demonstrate that a fraction of the surface neat carboxylic groups remained carboxylates, silicon originated from silica and PFOTES, whereas fluorine was exclusively from PFOTES. These results provided further confirmation that silica NPs and PFOTES were on the MNOCM surface.

The sizes of amino- $\mathrm{SiO}_{2} \mathrm{NPs}$ were varied for the covalent layer-by-layer assembly on the MNOCM surface to interrogate how particles sizes affect hydrophobicity (Table 2). Although, the $70 \mathrm{~nm}$ and $260 \mathrm{~nm}$ amino- $\mathrm{SiO}_{2} \mathrm{NPs}_{\text {showed }}$ superhydrophobicity, applying $500 \mathrm{~nm}$ ( $\sim$ twice the second sample size) amino- $\mathrm{SiO}_{2}$, the water contact angle was attenuated to $140^{\circ} \pm 2^{\circ}$. Therefore, the hydrophobicity of the raspberry-like structure for the silica NP coated surfaces is not only a function of the numbers of layers, but also of the size of the silica nanoparticles [1]. Surface roughness is likely more critical than surface chemical composition in determining hydrophobicity of such surfaces [1] [41].
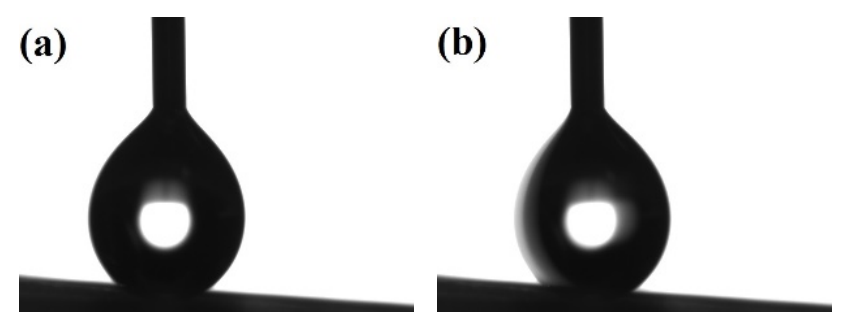

(c)
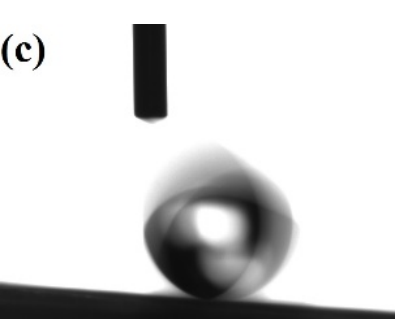

(d)

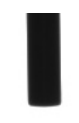

Figure 8 . The sliding process for a water droplet $(8 \mu \mathrm{L})$ on the MNOCM. 


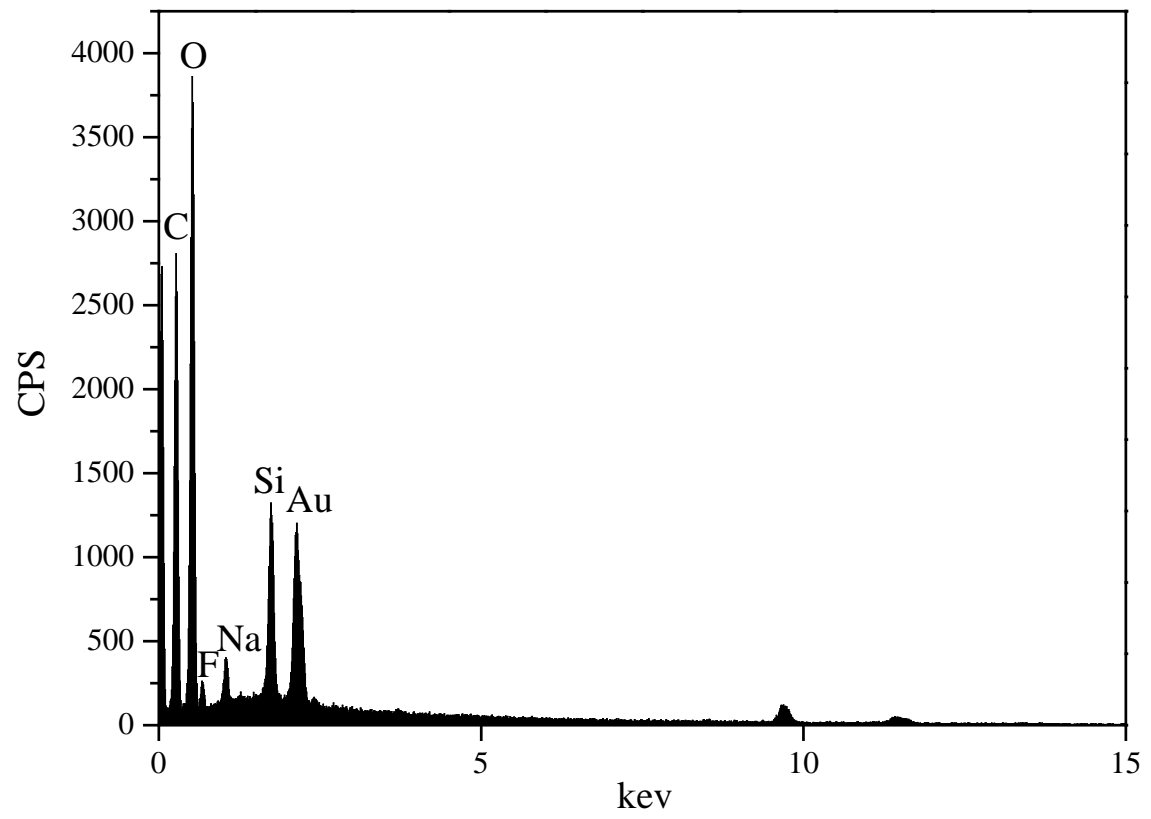

Figure 9. EDX spectra for the as-prepared super-hydrophobic MNOCM film.

Table 2. The water contact angles (WCA) of the surface with different sizes of the $\mathrm{SiO}_{2}$ nanoparticles after assembly of 5 layers.

\begin{tabular}{cccc}
\hline Samples & Amino-SiO$_{2}(\mathrm{~nm})$ & Epoxy-SiO$_{2}(\mathrm{~nm})$ & Water contact angle $\pm 2^{\circ}$ \\
\hline 1 & 70 & 30 & 150 \\
2 & 260 & 30 & 151 \\
3 & 500 & 30 & 140 \\
\hline
\end{tabular}

Self-cleaning is a property that is typically associated with superhydrophobicity as evidenced in the self-cleaning of mud from lotus leaves. Therefore, the self-cleaning of superhydrophobic MNOCM was evaluated according to a simple construct shown in Figure 10. The superhydrophobic-MNOCM films were attached to a glass slide onto which a dry slurry of activated carbon was placed to simulate contaminants (Figure 10(a)). Upon the disposition of water to the surface, the intrinsic surface tension of water on such an ultralow energy surface caused it to roll along down the incline due to gravity. During its roll, the contaminants on the surface were adsorbed (Figures 10(b)-10(e)), leaving a clean surface without any ostensible trace of contaminants (Figure 10(f)).

\section{Conclusion}

Superhydrophobic surfaces on MNOCM were successfully prepared via covalent LbL assembly of amino- $\mathrm{SiO}_{2} \mathrm{NPs}$ and epoxy- $\mathrm{SiO}_{2} \mathrm{NPs}$. The amino- $\mathrm{SiO}_{2} \mathrm{NPs}$ were grafted onto the MNOCM surface through a condensation reaction between the amino functionalities of the $\mathrm{SiO}_{2} \mathrm{NPs}$ and the carboxyl functionalities of the MNOCM surface followed by covalent LbL assembly of epoxy-SiO ${ }_{2} \mathrm{NPs}$. The change in wettability was attributed to increases in final LbL layer surface 

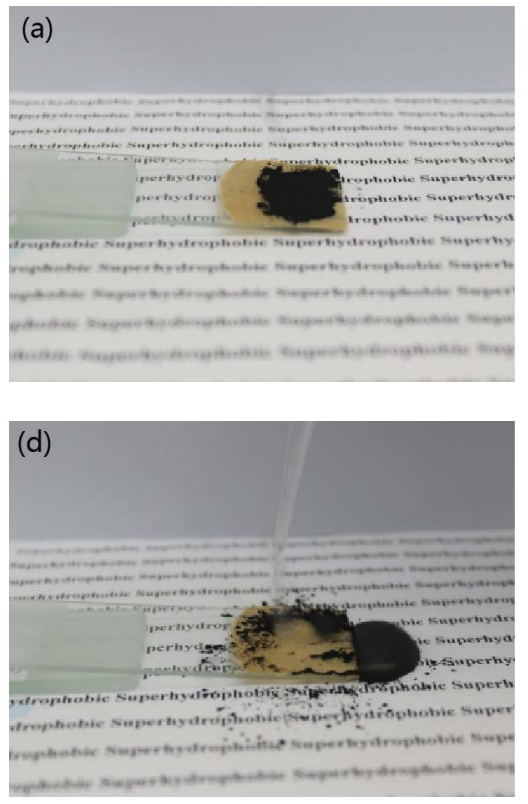
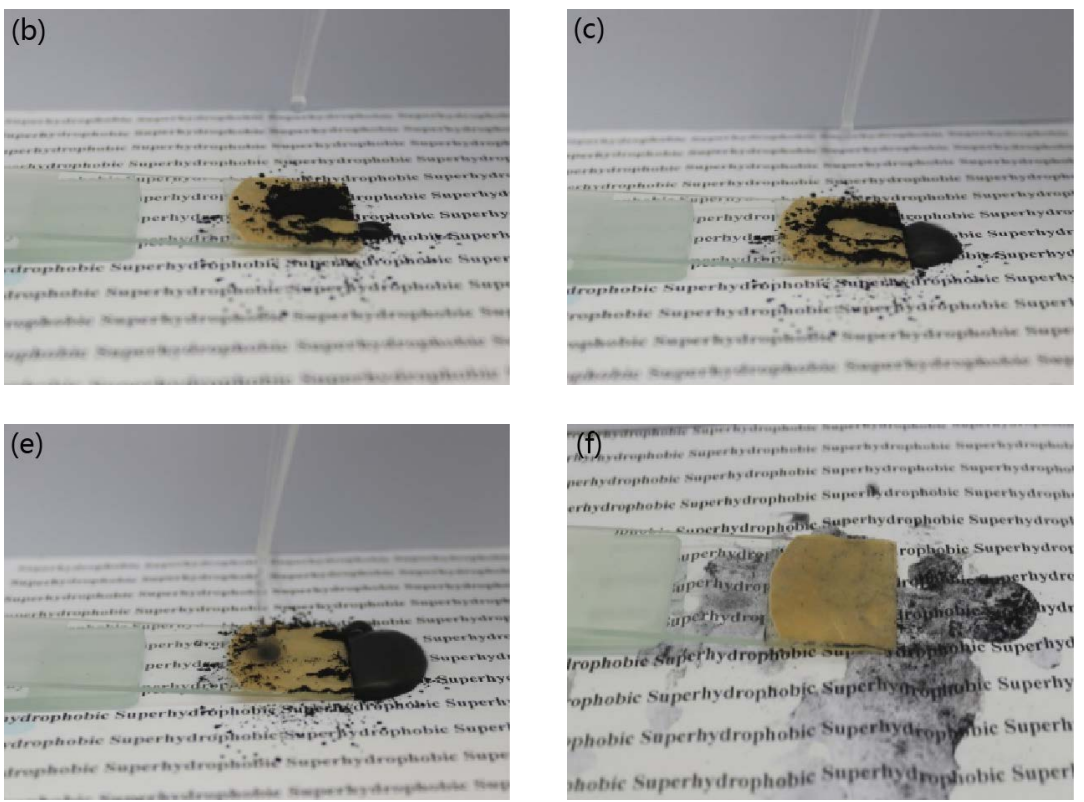

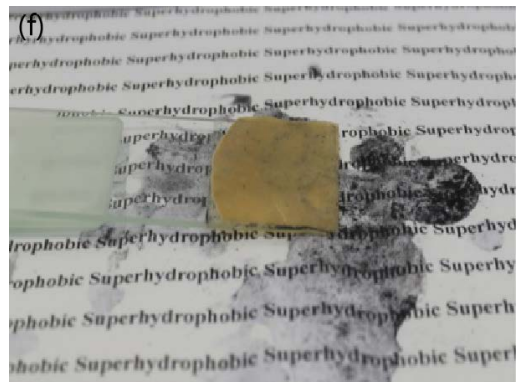

Figure 10. Simplified self-cleaning test for the superhydrophobic MNOCM films using a gently inclined glass slide upon which water droplets were added.

roughness induced by the sufficient LbL layering of alternating sizes of NPs akin to what is observed in a lotus leaf surface. Contact angle tests demonstrated that the obtained MNOCM displayed superhydrophobicity and good self-cleaning.

\section{Acknowledgements}

The work was generously supported by the Guangdong-Hongkong Joint Innovation Program (2014B050505019), the Natural Science Foundation of Guangdong Province, China (2014A030311030), the key project of the Science and Technology Ministry of Guangzhou, China (201607020025) and National Natural Science Foundation of China (31570569). The Authors also wish to also acknowledge the financial support awarded to LAL by the State Key Laboratory to guide the development of this work.

\section{References}

[1] Athauda, T.J., Williams, W., Roberts, K.P. and Ozer, R.R. (2013) On the Surface Roughness and Hydrophobicity of Dual-Size Double-Layer Silica Nanoparticles. Journal of Materials Science, 48, 6115-6120. https://doi.org/10.1007/s10853-013-7407-5

[2] Amigoni, S., De Givenchy, E.T., Dufay, M. and Guittard, F. (2009) Covalent Layer-by-Layer Assembled Superhydrophobic Organic-Inorganic Hybrid Films. Langmuir, 25, 11073-11077. https://doi.org/10.1021/la901369f

[3] Zang, D., Liu, F., Zhang, M., Niu, X., Gao, Z. and Wang, C. (2015) Superhydrophobic Coating on Fiberglass Cloth for Selective Removal of Oil from Water. Chemical Engineering Journal, 262, 210-216.

https://doi.org/10.1016/j.cej.2014.09.082

[4] She, Z., Li, Q., Wang, Z., Li, L., Chen, F. and Zhou, J. (2013) 228, 415. 
[5] Xue, C.H., Jia, S.T., Zhang, J., Tian, L.Q. and Chen, H.Z. (2008) SCI TECHNOL ADVMAT.

[6] Obeso, C.G., Sousa, M.P., Song, W., Rodriguez-Pérez, M.A., Bhushan, B. and Mano, J.F. (2013) Modification of Paper Using Polyhydroxybutyrate to Obtain Biomimetic Superhydrophobic Substrates. Colloids and Surfaces A: Physicochemical and Engineering Aspects, 416, 51-55. https://doi.org/10.1016/j.colsurfa.2012.09.052

[7] Sousa, M.P. and Mano, J.F. (2013) Superhydrophobic Paper in the Development of Disposable Labware and Lab-on-Paper Devices. ACS Applied Materials \& Interfaces, 5, 3731-3737. https://doi.org/10.1021/am400343n

[8] Zhou, Z. and Wu, X.F. (2015) Electrospinning Superhydrophobic-Superoleophilic Fibrous PVDF Membranes for High-Efficiency Water-Oil Separation. Materials Letters, 160, 423-427. https://doi.org/10.1016/j.matlet.2015.08.003

[9] Wang, S., Peng, X., Zhong, L., Tan, J., Jing, S., Cao, X., Chen, W., Liu, C. and Sun, R. (2015) An Ultralight, Elastic, Cost-Effective, and Highly Recyclable Superabsorbent from Microfibrillated Cellulose Fibers for Oil Spillage Cleanup. Journal of Materials Chemistry A, 3, 8772. https://doi.org/10.1039/C4TA07057G

[10] Yokoi, N., Manabe, K., Tenjimbayashi, M. and Shiratori, S. (2015) Optically Transparent Superhydrophobic Surfaces with Enhanced Mechanical Abrasion Resistance Enabled by Mesh Structure. ACS Applied Materials \& Interfaces, 7, 4809-4816. https://doi.org/10.1021/am508726k

[11] Jradi, K., Laour, D., Daneault, C. and Chabot, B. (2011) Control of the Chemical and Physical Behaviour of Silicon Surfaces for Enhancing the Transition from Hydrophilic to Superhydrophobic Surfaces. Colloids and Surfaces A: Physicochemical and Engineering Aspects, 374, 33-41. https://doi.org/10.1016/j.colsurfa.2010.10.050

[12] Li, X., Hu, D., Huang, K. and Yang, C. (2014) Hierarchical Rough Surfaces Formed by LBL Self-Assembly for Oil-Water Separation. Journal of Materials Chemistry A, 2, 11830-11838. https://doi.org/10.1039/C4TA01569J

[13] Li, H., Yang, J., Li, P., Lan, T. and Peng, L. (2017) A Facile Method for Preparation Superhydrophobic Paper with Enhanced Physical Strength and Moisture-Proofing Property. Carbohydrate Polymers, 160, 9-17. https://doi.org/10.1016/j.carbpol.2016.12.018

[14] García, B., Saiz-Poseu, J., Gras-Charles, R., Hernando, J., Alibés, R., Novio, F., Sedó, J., Busqué, F. and Ruiz-Molina, D. (2014) Mussel-Inspired Hydrophobic Coatings for Water-Repellent Textiles and Oil Removal. ACS Applied Materials \& Interfaces, 6, 17616-17625. https://doi.org/10.1021/am503733d

[15] Leng, B., Shao, Z., de With, G. and Ming, W. (2009) Superoleophobic Cotton Textiles. Langmuir, 25, 2456-2460. https://doi.org/10.1021/la8031144

[16] Gao, Z., Zhai, X., Liu, F., Zhang, M., Zang, D. and Wang, C. (2015) Fabrication of $\mathrm{TiO}_{2} /$ EP Super-Hydrophobic Thin Film on Filter Paper Surface. Carbohydrate Polymers, 128, 24-31. https://doi.org/10.1016/j.carbpol.2015.04.014

[17] Nooy, A., Besemer, A.C. and Bekkum, H.V. (1995) Highly Selective Nitroxyl Radical-Mediated Oxidation of Primary Alcohol Groups in Water-Soluble Glucans. CARBOHYD RES, 269, 89-98. https://doi.org/10.1016/0008-6215(94)00343-E

[18] Saito, T., Nishiyama, Y., Putaux, J., Vignon, M. and Isogai, A. (2006) Homogeneous Suspensions of Individualized Microfibrils from TEMPO-Catalyzed Oxidation of Native Cellulose. Biomacromolecules, 7, 1687-1691. https://doi.org/10.1021/bm060154s

[19] Isogai, A., Saito, T. and Fukuzumi, H. (2011) TEMPO-Oxidized Cellulose Nanofibers. Nanoscale, 3, 71-85. https://doi.org/10.1039/C0NR00583E 
[20] Saito, T. and Isogai, A. (2007) Wet Strength Improvement of TEMPO-Oxidized Cellulose Sheets Prepared with Cationic Polymers. Industrial \& Engineering Chemistry Research, 46, 773-780. https://doi.org/10.1021/ie0611608

[21] Fukuzumi, H., Saito, T., Iwata, T., Kumamoto, Y. and Isogai, A. (2009) Transparent and High Gas Barrier Films of Cellulose Nanofibers Prepared by TEMPO-Mediated Oxidation. Biomacromolecules, 10, 162-165. https://doi.org/10.1021/bm801065u

[22] Tang, H., Butchosa, N. and Zhou, Q. (2015) A Transparent, Hazy, and Strong Macroscopic Ribbon of Oriented Cellulose Nanofibrils Bearing Poly(ethylene glycol) Advanced Materials, 27, 2070-2076. https://doi.org/10.1002/adma.201404565

[23] Saito, T. and Isogai, A. (2005) Novel Method to Improve Wet Strength of Paper. TAPPI Journal, 4, 3-8.

[24] Saito, T. and Isogai, A. (2006) Introduction of Aldehyde Groups on Surfaces of Native Cellulose Fibers by TEMPO-Mediated Oxidation. Colloids \& Surfaces: A Physicochemical \& Engineering Aspects, 289, 219-225.

[25] Meng, Q., Li, H., Fu, S. and Lucia, L.A. (2014) The Non-Trivial Role of Native Xylans on the Preparation of TEMPO-Oxidized Cellulose Nanofibrils. Reactive and Functional Polymers, 85, 142-150. https://doi.org/10.1016/j.reactfunctpolym.2014.07.021

[26] Ber, W.S., Fink, A. and Bohn, E. (1968) Controlled Growth of Monodisperse Silica Spheres in the Micron Size Range. Journal of Colloid and Interface Science, 26, 62-69.

[27] Ming, W., Wu, D., van Benthem, R. and de With, G. (2005) Superhydrophobic Films from Raspberry-Like Particles. Nano Letters, 5, 2298-2301. https://doi.org/10.1021/nl0517363

[28] Stéphane, R., Poncetlegrand, C., Ravaine, S., Mingotaud, C. and Duguet, A.E. (2002) Syntheses of Raspberrylike Silica/Polystyrene Materials. Chemistry Materials, 14, 2354-2359. https://doi.org/10.1021/cm0116525

[29] Erbil, H.Y., Demirel, A.L., Avc1, Y. and Mert, O. (2003) Transformation of a Simple Plastic into a Superhydrophobic Surface. Science, 299, 1377-1380.

https://doi.org/10.1126/science.1078365

[30] Stöber, W., Fink, A. and Bohn, E. (1968) Controlled Growth of Monodisperse Silica Spheres in the Micron Size Range. Journal of Colloid \&amp; Interface Science, 26, 62-69. https://doi.org/10.1016/0021-9797(68)90272-5

[31] Zhang, M. and Wang, C. (2013) Fabrication of Cotton Fabric with Superhydrophobicity and Flame Retardancy. Carbohydrate Polymers, 96, 396-402. https://doi.org/10.1016/j.carbpol.2013.04.025

[32] Wang, R., Bao, S., Liu, F., Jiang, X., Zhang, Q., Sun, B. and Zhu, M. (2013) Wear Behavior of Light-Cured Resin Composites with Bimodal Silica Nanostructures as Fillers. Materials Science and Engineering C, 33, 4759-4766. https://doi.org/10.1016/j.msec.2013.07.039

[33] Fujisawa, S., Okita, Y. and Fukuzumi, H. (2011) Preparation and Characterization of TEMPO-Oxidized Cellulose Nanofibril Films with Free Carboxyl Groups. Carbohydrate Polymers, 84, 579-583. https://doi.org/10.1016/j.carbpol.2010.12.029

[34] Nge, T.T., Nogi, M., Yano, H. and Sugiyama, J. (2010) Microstructure and Mechanical Properties of Bacterial Cellulose/Chitosan Porous Scaffold. Cellulose, 17, 349-363. https://doi.org/10.1007/s10570-009-9394-x

[35] Staroszczyk, H., Pielichowska, J., Sztuka, K., Stangret, J. and Odziejska, I.K. (2012) Molecular and Structural Characteristics of Cod Gelatin Films Modified with EDC and TGase. Food Chemistry, 130, 335-343. https://doi.org/10.1016/j.foodchem.2011.07.047 
[36] Ayutsede, J., Gandhi, M., Sukigara, S., Micklus, M., Chen, H. and Ko, F. (2005) Regeneration of Bombyx mori Silk by Electrospinning. Part 3: Characterization of Electrospun Nonwoven Mat. Polymer, 46, 1625-1634.

https://doi.org/10.1016/j.polymer.2004.11.029

[37] Lavorgna, M., Romeo, V., Martone, A., Zarrelli, M., Giordano, M., Buonocore, G.G., Qu, M.Z., Fei, G.X. and Xia, H.S. (2013) Silanization and Silica Enrichment of Multiwalled Carbon Nanotubes: Synergistic Effects on the Thermal-Mechanical Properties of Epoxy Nanocomposites. European Polymer Journal, 49, 428-438. https://doi.org/10.1016/j.eurpolymj.2012.10.003

[38] Hao, L., An, Q. and Xu, W. (2012) Facile fabrication of Superhydrophobic Cotton Fabric from Stearyl Methacrylate Modified Polysiloxane/Silica Nanocomposite. Fibers and Polymers, 13, 1145-1153. https://doi.org/10.1007/s12221-012-1145-1

[39] Saito, T. and Isogai, A. (2004) TEMPO-Mediated Oxidation of Native Cellulose. The Effect of Oxidation Conditions on Chemical and Crystal Structures of the Water-Insoluble Fractions. Biomacromolecules, 5, 1983-1989.

https://doi.org/10.1021/bm0497769

[40] Li, X. and He, J. (2012) In situ Assembly of Raspberry- and Mulberry-Like Silica Nanospheres toward Antireflective and Antifogging Coatings. ACS Applied Materials \& Interfaces, 4, 2204-2211. https://doi.org/10.1021/am3002082

[41] Athauda, T.J., Decker, D.S. and Ozer, R.R. (2012) Effect of Surface Metrology on the Wettability of $\mathrm{SiO}_{2}$ Nanoparticle Coating. Materials Letters, 67, 338-341. https://doi.org/10.1016/j.matlet.2011.09.100 